\title{
Sparsity Inspired Selection And Recognition Of Iris Images
}

\author{
Jaishanker K. Pillai, Vishal M. Patel and Rama Chellappa
}

\begin{abstract}
Iris images acquired from a partially cooperating subject often suffer from blur, occlusion due to eyelids, and specular reflections. The performance of existing iris recognition systems degrade significantly on these images. Hence it is essential to select good images from the incoming iris video stream, before they are input to the recognition algorithm. In this paper, we propose a sparsity based algorithm for selection of good iris images and their subsequent recognition. Unlike most existing algorithms for iris image selection, our method can handle segmentation errors and a wider range of acquisition artifacts common in iris image capture. We perform selection and recognition in a single step which is more efficient than devising separate specialized algorithms for the two. Recognition from partially cooperating users is a significant step towards deploying iris systems in a wide variety of applications.
\end{abstract}

\section{INTRODUCTION}

Iris recognition is one of the most promising approaches for biometric authentication. The iris pattern is unique to each individual and is believed to remain essentially stable through out ones life time. Existing algorithms based on extracting and matching features from the iris have reported very high recognition rates on clean iris datasets. Since they rely on the fine textural features on the human iris for recognition, their performance will degrade significantly when the image quality is poor. Iris segmentation results will also be poor on such images, which will in turn affect the recognition rate. So most of them require good quality iris images which in turn demands high level of cooperation of the users.

This seriously limits the application of iris recognition systems in uncontrolled scenarios, where user cooperation is normally limited. When the subjects are not fully cooperating with the system, many of the acquired iris images will suffer from artifacts such as defocus blur, motion blur, occlusion due to eyelids and specular reflections on the iris. Fig. 1 shows some of these distortions on images from the ND-IRIS-0405 dataset [1]. Hence it is essential to first select the "recognizable" iris images before employing the recognition algorithm. In this paper, we extend the use of sparse representations for recognition as proposed in [2], for selection and recognition of iris images with a modified cost function. The chief novelties of our algorithm are

1) We have utilized sparse representations techniques for iris image selection and recognition.

This work was supported by MURI from the Office of Naval Research under the Grant N00014_08_I_0638

Authors are with the Department Of Electrical and Computer Engineering and the Center for Automation Research, UMIACS, University of Maryland, College Park, MD 20742 $\{j \mathrm{sp}$, pvishalm,Rama\}@umiacs.umd.edu
2) Our method can perform both iris selection and recognition in a single step, which is more efficient than utilizing two separate algorithms for the same.

3) We can handle a wide variety of acquisition artifacts such as blur, occlusion and specular reflections.

4) The proposed image quality measure can handle segmentation errors unlike most of the existing methods. This is a very useful asset as segmentation results will also be poor when the image quality is bad.

5) The introduced quality measure is also a confidence measure of recognition as demonstrated in section VE.

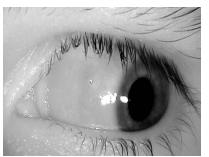

(a)

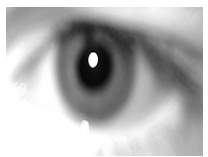

(b)

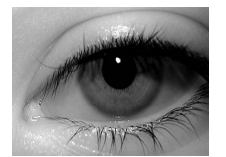

(c)

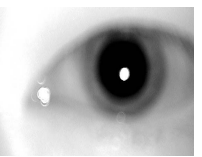

(d)
Fig. 1. Some badly acquired iris images from the ND-IRIS-0405 dataset. Note that image (a) has specular reflections on the iris and is also hard to be segmented correctly due to the tilt and non circular shape. Images (b) and (d) are blurred whereas image (c) is occluded by the shadow of the eyelids.

The organization of the paper is as follows. In section II, we discuss some of the existing algorithms for iris image selection and recognition. The theory of sparse representation is presented in section III. Section IV describes our algorithm. We present experiments and results on simulated and real iris images in section $\mathrm{V}$. We briefly outline the future work and conclude the paper in section VI.

\section{BACKGROUND}

The first operational iris system was developed by Daugman [3] in 1993. In his method, Gabor features were extracted from scale normalized iris regions and were quantized to form a $2 \mathrm{~K}$ bit iris code. The Hamming distance between the iris code of the test and the training iris images was used for recognition. Wildes [4] used Laplacian of Gaussian filter at multiple scales to produce a template and used the normalized correlation as the similarity measure. Numerous iris recognition algorithms have been proposed in recent years. Most of them differ from Daugman's algorithm in the image feature selection and matching scheme they use. See [5] for an excellent survey of papers on iris recognition. Most of these algorithms assume good quality iris images and perfect segmentation, which is not possible in an iris recognition system without full cooperation from the users. 
Good quality images are essential for biometric systems [6]. Daugman [3] used the energy of the high frequency components as a measure of blur. Proenca and Alexandre [7] trained a neural network to identify common noise degradations in iris images. Zhu et al. [8] used the wavelet coefficients to evaluate the quality of the iris images. The Fourier spectra of local iris regions was used by Ma et al. to characterize blur and occlusion [9]. Rakshit and Monro used the quality and position of specular reflections for selecting good quality images [10]. A statistical method to detect wide variety of artifacts is presented in [11]. All these methods assume good iris segmentation. But iris segmentation will be equally poor when the image quality is bad. Chen et al. [12] proposed image quality estimation without assuming good segmentation. Most of these algorithms are specialized for image selection and so a separate method is required for recognizing iris images with an exception of the Daugman's method. This will be slower and also computationally more demanding. Majority of the methods above use some heuristics to measure iris image quality and hence cannot handle the wide variety of common artifacts possible during iris image acquisition. In contrast, the introduced image quality measure can handle segmentation errors, occlusion, specular reflections and blur. We also perform selection and recognition in a single step.

A parallel area of research aimed at reducing the user co-operation in iris recognition systems is developing less intrusive acquisition systems. One significant work is the "Iris on the Move" project by Matey et al. [13], which can capture iris images over a wider volume. Wheeler et al. developed a standoff iris recognition system, which can recognize people standing in front and facing the system [14]. Their approach is to "continuously capture iris images and attempt recognition until it succeeds". We believe that using an image selection algorithm like ours will further improve the performance of these systems.

\section{THEORY}

In object recognition, given a set of labeled training samples, the task is to identify the class to which a test sample belongs to. In what follows, we propose to use sparse representations of images for recognition of iris images.

\section{A. Sparse Representations}

Suppose that we are given $L$ distinct classes and a set of $n$ training images per class. We identify an $l \times p$ grayscale image as an $N$-dimensional vector which can be obtained by stacking its columns. Let $\mathbf{D}_{k}=\left[\mathbf{x}_{k 1}, \ldots, \mathbf{x}_{k n}\right]$ be an $N \times n$ matrix of training images from the $k^{\text {th }}$ class. Define a new matrix, $\mathbf{D}$, as the concatenation of training samples from all the classes as

$$
\begin{aligned}
\mathbf{D} & =\left[\mathbf{D}_{1}, \ldots, \mathbf{D}_{L}\right] \in \mathbb{R}^{N \times(n . L)} \\
& =\left[\mathbf{x}_{11}, \ldots, \mathbf{x}_{1 n}\left|\mathbf{x}_{21}, \ldots, \mathbf{x}_{2 n}\right| \ldots \ldots \mid \mathbf{x}_{L 1}, \ldots, \mathbf{x}_{L n}\right]
\end{aligned}
$$

We consider an observation vector $\mathbf{y} \in \mathbb{R}^{N}$ of unknown class as a linear combination of the training vectors as

$$
\mathbf{y}=\sum_{i=1}^{L} \sum_{j=1}^{n} \alpha_{i j} \mathbf{x}_{i j}
$$

with coefficients $\alpha_{i j} \in \mathbb{R}$. The above equation can be more compactly written as

$$
\mathbf{y}=\mathbf{D} \alpha
$$

where

$$
\alpha=\left[\alpha_{11}, \ldots, \alpha_{1 n}\left|\alpha_{21}, \ldots, \alpha_{2 n}\right| \ldots \ldots \mid \alpha_{L 1}, \ldots, \alpha_{L n}\right]^{T}
$$

and.$^{T}$ denotes the transposition operation. Here, we make an assumption that given sufficient training samples of the $k^{t h}$ class, $\mathbf{D}_{k}$, any new test image $\mathbf{y} \in \mathbb{R}^{N}$ that belongs to the same class will approximately lie in the linear span of the training samples from the class $k$. This implies that most of the coefficients not associated with class $k$ in (3) will be close to zero.

\section{B. Sparse Recovery}

In order to represent an observed vector $\mathbf{y} \in \mathbb{R}^{N}$ as a sparse vector $\alpha$, one needs to solve the system of linear equations (2). There exists several decomposition methods for obtaining solutions to (2). One such method is known as the Method of Frames (MOF) [15]. Among all the solutions of (2), the MOF picks out the one whose coefficients have minimum $\ell_{2}$ norm

$$
\hat{\alpha}=\arg \min _{\alpha^{\prime} \in \mathbb{R}^{N}}\left\|\alpha^{\prime}\right\|_{2} \text { subject to } \mathbf{y}=\mathbf{D} \alpha^{\prime} .
$$

The solution to the above problem is given by

$$
\hat{\alpha}=\mathbf{D}^{\dagger} \mathbf{y}=\mathbf{D}^{*}\left(\mathbf{D D}^{*}\right)^{-1} \mathbf{y},
$$

where $\mathbf{D}^{*}$ is the adjoint of $\mathbf{D}$. This solution, however, yields a non-sparse vector which may not be informative for the recognition task.

The approach, we take in this paper, is to instead find the sparsest solution to $\mathbf{y}=\mathbf{D} \alpha$. This can be done by solving the following optimization problem

$$
\hat{\alpha}=\arg \min _{\alpha^{\prime} \in \mathbb{R}^{N}}\left\|\alpha^{\prime}\right\|_{1} \quad \text { subject to } \mathbf{y}=\mathbf{D} \alpha^{\prime},
$$

where $\|\cdot\|_{1}$ denotes the $\ell_{1}$ norm. This problem is often known as Basis Pursuit (BP) and can be solved in polynomial time [15]. In the case when noisy observations are given, Basis Pursuit DeNoising (BPDN) can be used to estimate $\alpha$

$$
\hat{\alpha}=\arg \min _{\alpha^{\prime} \in \mathbb{R}^{N}}\left\|\alpha^{\prime}\right\|_{1} \quad \text { subject to }\left\|\mathbf{y}-\mathbf{D} \alpha^{\prime}\right\| \leq \varepsilon,
$$

where we have assumed the observation are of the form $\mathbf{y}=\mathbf{D} \alpha+\eta$ with $\|\eta\|_{2} \leq \varepsilon$. 


\section{Sparse Recognition}

Given an observation vector $\mathbf{y}$ from one of the $L$ classes in the training set, we compute its coefficients $\hat{\alpha}$ by solving either (6) or (7). We perform classification based on the fact that high values of the coefficients $\hat{\alpha}$ will be associated with the columns of $\mathbf{D}$ from a single class. We do this by comparing how well the different parts of the estimated coefficients, $\hat{\alpha}$, represent $\mathbf{y}$. The minimum of the representation error or the residual error is then used to identify the correct class. The residual error of class $k$ is calculated by keeping the coefficients associated with that class and setting the coefficients not associated with class $k$ to zero. This can be done by introducing a characteristic function, $\Pi_{k}$, that selects the coefficients associated with the $k^{\text {th }}$ class as follows

$$
r_{k}(\mathbf{y})=\left\|\mathbf{y}-\mathbf{D} \Pi_{k}(\hat{\alpha})\right\|_{2} .
$$

The class, $d$, that is associated to an observed vector, is then declared as the one that produces the smallest approximation error

$$
d=\arg \min _{k} r_{k}(\mathbf{y}) .
$$

We now summarize our algorithm as follows:

Given a matrix of training samples $\mathbf{D} \in \mathbb{R}^{N \times(n . L)}$ for $L$ classes and a test sample $\mathbf{y} \in \mathbb{R}^{N}$ :

1) Solve the BP (6) or BPDN (7) problem.

2) Compute the residual using (8).

3) Identify y using (9).

Instead of minimizing either (6) or (7) as done in [2], we minimize the following modified BPDN problem

$$
\hat{\alpha}=\arg \min _{\alpha^{\prime} \in \mathbb{R}^{N}}\|\mathbf{z}\|_{1} \quad \text { subject to }\left\|\mathbf{y}-\mathbf{D} \alpha^{\prime}\right\| \leq \varepsilon
$$

where $\mathbf{z}=\left[z_{1}, \ldots, z_{L}\right] \in \mathbb{R}^{L}$ and $z_{k}=\left\|\Pi_{k}\left(\alpha^{\prime}\right)\right\|_{2}$. By minimizing the L1 norm of $\mathbf{z}$, we enforce the training vectors of the same class to have high coefficients, thereby producing sparser vectors in contrast to the method proposed in [2].

\section{Image quality measure}

For classification, it is important to be able to detect and then reject invalid test samples. To decide whether a given test sample is a valid sample or not, the notion of Sparsity Concentration Index $(S C I)$ was proposed in [2]. The $S C I$ of a coefficient vector $\alpha \in \mathbb{R}^{(L . n)}$ is defined as

$$
\operatorname{SCI}(\alpha)=\frac{\frac{L \cdot \max \left\|\Pi_{i}(\alpha)\right\|_{1}}{\|\alpha\|_{1}}-1}{L-1} .
$$

$S C I$ takes values between 0 and 1 . $S C I$ values close to 1 correspond to the fact that the test image can be approximately represented by using only images from a single class. If $S C I=0$ then the coefficients are spread evenly over all classes. A threshold can be chosen such that it rejects outliers. For instance, a test image can be rejected if $S C I(\hat{\alpha})<\lambda$ and otherwise accepted as valid, where $\lambda$ is some chosen threshold between 0 and 1 .

\section{IRIS SELECTION AND RECOGNITION}

In this section, we describe various steps of our algorithm. The iris image is first segmented using Hough Transforms [16]. In order to retain realistic segmentation errors possible in a practical scenario, we have avoided manual segmentation of the wrongly segmented images. From the segmented iris image, a $20 \times 240$ iris template is formed by sampling. The Gabor features of the template of the training images are used to populate the $\mathbf{D}$ matrix in section III-C. Note that our algorithm is independent of the features used and one can combine easily multiple features to improve the performance. Corresponding to each test image, the $\mathbf{y}$ vector is formed from the iris template in the same manner. In our approach, we employed a highly efficient algorithm that is suitable for large scale applications known as the Spectral Projected Gradient (SPGL1) algorithm [17] to obtain the sparse representation. From the representation, the SCI is computed using equation (11) and is compared to a predefined threshold to remove the poor quality images. The block diagram of our method is given in Fig. 2 .

To speed up our algorithm, we have performed recognition separately for different sectors of the iris and combined the individual results by voting. This can be done in parallel and also makes our algorithm robust to occlusions and specular reflections.

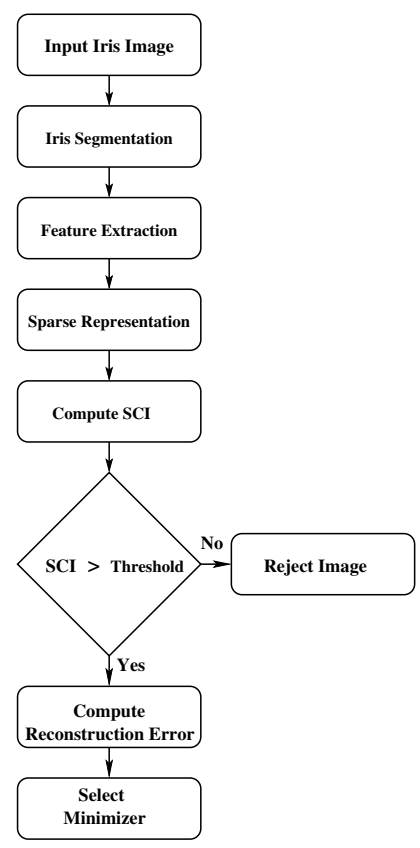

Fig. 2. Block diagram of the proposed method.

\section{EXPERIMENTS AND RESULTS}

In the following subsections, we present image selection and recognition results on simulated data as well as on real images from the ND-IRIS-0405 dataset [1]. It is a superset of the ICE2005 and ICE2006 iris datasets. It contains about 65,000 iris images belonging to 356 persons, with a wide variety of distortions, facilitating the testing and performance 
evaluation of our algorithm. To illustrate the robustness of our algorithm to occlusion due to eyelids and eyelashes, we perform only a simple iris segmentation scheme detecting just the pupil and iris boundaries and not the segmentation of the eyelids and eye lashes. We use the publically available code of Masek et al. [16] for detecting these boundaries.

\section{A. Variation of SCI with common distortions during image acquisition}

Some of the common distortions possible during iris image acquisition are blurring due to defocus and movement of the subject, occlusion due to eye lids and specular reflections. There can also be errors in iris segmentation. To study the variation in SCI in the presence of these distortions, we simulate them on the clean iris images from the ND dataset. We selected the clean iris images of the left eye of eighty persons. Ten such images per person formed the gallery and distortions were simulated on the remaining images, which formed the probes. We consider seven different levels of distortion for each case, with level one indicating no distortion and level seven indicating maximum distortion.

Fig. 3 shows some of the simulated images from the ND dataset. The first column includes images with distortion level one (no distortion). The middle column contains images with distortion level three (moderate distortions). The right most column contain images with distortion level five (high distortion). The first row contains images with blur while the second contains images with occlusion. Images with simulated segmentation error and specular reflections are shown in the third and fourth rows respectively.

Fig. 4 illustrates the variation of SCI with the common acquisition distortions. It can be observed that good images have high SCI values whereas the ones with distortion have lower SCI values. So by suitably thresholding the SCI value of the test image, we can remove the bad images before the recognition stage. Observe that the relatively lower decrease in SCI with occlusion and specular reflection explains the increased robustness attained by our algorithm through separate recognition and voting described in section IV.

\section{B. Image Selection Results on the ND Dataset}

In this section, we illustrate the performance of our image selection algorithm on the images from the ND dataset. We selected 80 persons having sufficiently large number of iris images with different distortions like blur, occlusion and segmentation errors. Fifteen clean images per person were hand picked which formed the gallery. Upto fifteen images with blur, occlusion and segmentation errors were also selected. As mentioned above, we perform just a simple segmentation scheme, retaining possible occlusion due to eyelids and eyelashes in the iris vector. The Gabor features of the iris vector are computed and are input to our algorithm.

Fig. 5(a) shows the Receiver Operator Characteristics(ROC) of our algorithm (black), compared to the publically available iris recognition system of Masek et al. [16] (red), when the probe images are blurred. Since the data has occlusion, for a fair comparison, we modified his algorithm,

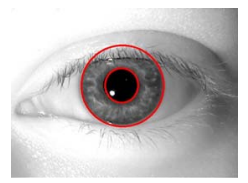

(a)

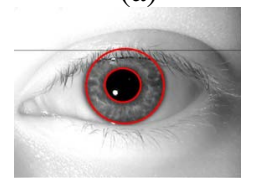

(d)

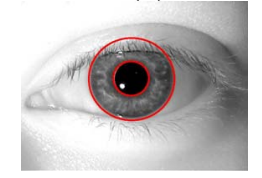

$(\mathrm{g})$

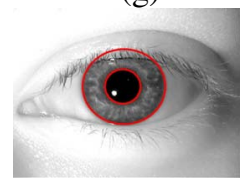

(j)

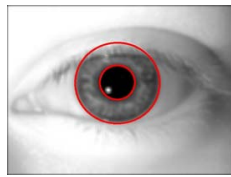

(b)

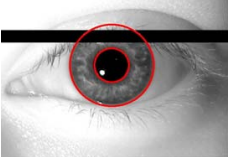

(e)

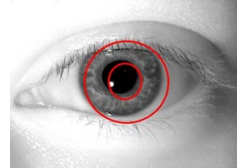

(h)

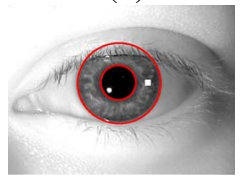

(k)

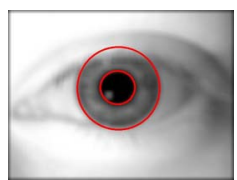

(c)

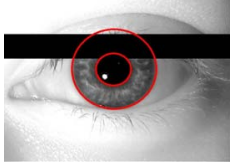

(f)

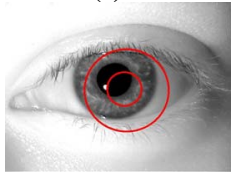

(i)

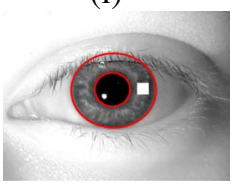

(1)
Fig. 3. Simulated Distortions on the images from the ND dataset. The detected pupil and iris boundaries are indicated as red circles.

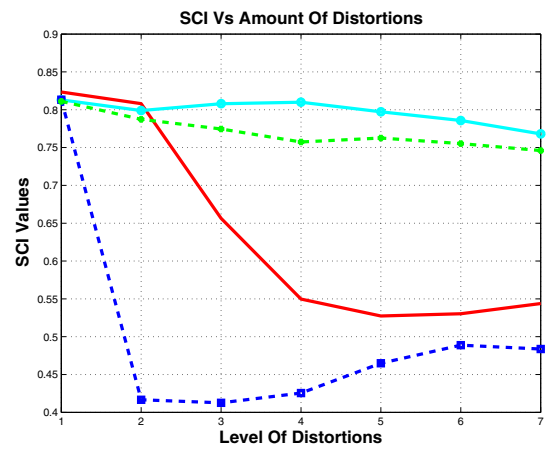

Fig. 4. Plot of the variation in SCI values with common distortions in iris image acquisition. Red(Blur), Green(Specular Reflections), Blue (Segmentation Errors) and Cyan(Occlusion due to eyelids). Note that the SCI falls monotonically with deterioration in the iris images.

recognizing the different sectors of the iris separately and fusing the results through voting. Note that our ROC curve is significantly sharper than that of the Masek's recognition system indicating superior performance.

The effect of occlusion in iris images due to eye lids, eye lashes and specular reflections are illustrated in Fig. 5(b). Images with occlusion were obtained for each of the eighty classes under consideration and were used as probes. The ROC curve of our algorithm is shown in black and that of Masek's system is shown in red. Note that our method gives a higher verification rate when compared to the Masek's implementation for each false positive rate.

To study the effects of segmentation error, the gallery images were verified to be well segmented. Upto fifteen images with segmentation errors were chosen for each person under consideration, which formed the probes. Fig. 5(c) shows the 
ROC curves of our method (black) and the Masek's one (red) in case of wrongly segmented images. Again using our image selection algorithm improves the performance of the system even with wrongly segmented images, an asset which many of the existing quality estimation methods lack.

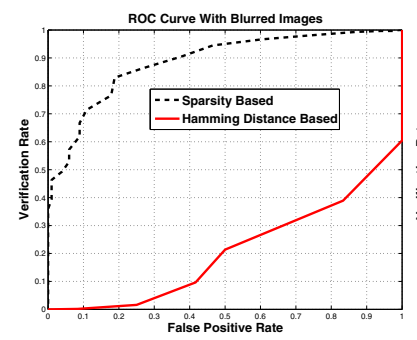

(a)

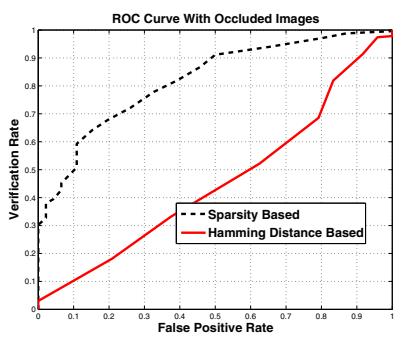

(b)

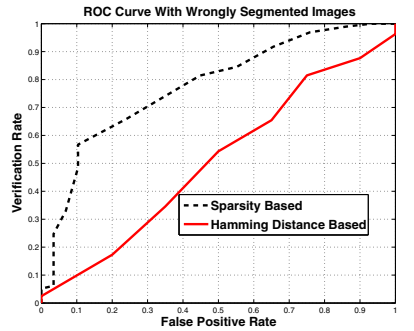

(c)

Fig. 5. Comparison of the ROC curves for our algorithm (Sparsity Based) and publically available algorithm of Masek (Hamming Distance Based) using clean iris images in the gallery and probe images containing (a) Blurring (b) Occlusions and (c) Segmentation Errors.

\section{Recognition Results On Images From The ND Dataset}

In this section, we first illustrate the performance of our recognition algorithm on clean images from the ND dataset. As explained before, eighty subjects were selected from the dataset. Fifteen clean images of the left iris were hand picked for each person. Out of these fifteen images per person, twelve were randomly selected to form the gallery and the remaining three images per person were used as probe. Since the dataset is clean, we avoided the image selection stage and directly applied our iris recognition algorithm. This enables us to evaluate the recognition performance of our algorithm separately. We compare our algorithm to a nearest neighbour based recognition algorithm(NN) on the Gabor features and Masek's implementation. Since we use tough segmentation conditions retaining the eye lids and eye lashes in the iris vector, direct application of NN and Masek's method gave poor results. For fair comparison, we divided the iris images into different sectors, obtained the results using these methods separately on each sectors and combined the results by voting. We obtained a recognition rate of $99.17 \%$ when compared to $98.33 \%$ for the $\mathrm{NN}$ and 97.5\% for the Masek's method.

To evaluate the recognition performance of our algorithm on poorly acquired images, we hand picked images with blur, occlusion and segmentation errors as explained in the previous section. 15 clean images per person were used to form the gallery. Probes containing each type of distortion were separately applied to the algorithm and the recognition rates are reported in Table. I. An SCI threshold of .7 was used in these experiments. In Fig. 6, we display the iris

TABLE I

RECOGNITION RATE ON ND DATASET

\begin{tabular}{||c||c||c||c||}
\hline Image Quality & NN & Masek's Implementation & Our Method \\
\hline Good & 98.33 & 97.5 & 99.17 \\
\hline Blured & 95.42 & 96.01 & 96.28 \\
\hline Occluded & 85.03 & 89.54 & 90.30 \\
\hline Seg. Error & 78.57 & 82.09 & 91.36 \\
\hline
\end{tabular}

images having the least SCI value for the blur, occlusion and segmentation error experiments performed on the real iris images in the ND dataset as mentioned above. As can be observed, the low SCI images suffer from high amounts of distortion.

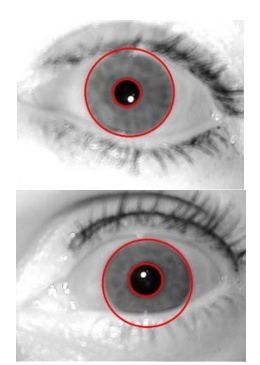

(a)

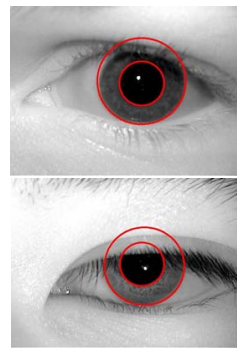

(b)

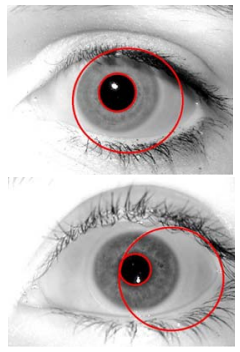

(c)
Fig. 6. Iris images with low SCI values in the ND dataset. Note that the images in (a), (b) and (c) suffer from high amounts of blur, occlusion and segmentation errors respectively .

\section{Effect of the number of training images on performance}

In this section, we study the effect of the number of training images on recognition rate of our algorithm. We vary the number of training images from one per class to twelve per class on the ND dataset. The testing images consisting of three iris images per person are used to test each of these cases. The variation of recognition rate obtained is plotted in Fig. 7. The more the number of training images, the better the recognition rate becomes. This is hardly surprising as our assumption that the training images span the space of testing images becomes more valid as the number of training images increases. In unconstrained iris recognition systems which we are interested in, this is not a bottle neck as we can get large number of iris images from the incoming iris video. So one can expect to have sufficiently high training images, even after removing the bad ones by our image selection algorithm.

\section{E. SCI as a measure of confidence in Recognition}

We have empirically observed that higher the SCI value for the test image, higher the probability that it is correctly classified. This is illustrated in Fig. 8. This observation is expected as high SCI means that the reconstructed vector will be sparse. If the training images span the space of possible 


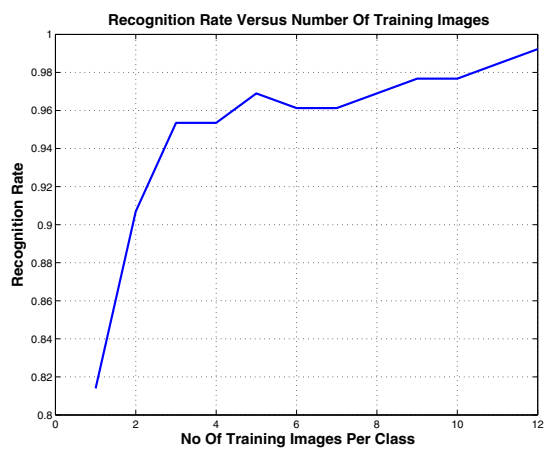

Fig. 7. Plot of the variation in recognition rate of our algorithm with the number of training images per class. Note that the performance increase monotonically with the number of training images.

testing images, the training images of the correct class will have high coefficients. So the only possible sparse vector is the one in which the correct class has high coefficients and others having zero coefficients, which will be correctly classified by our algorithm. So by putting a higher threshold on SCI during the image selection stage, we can reduce the false positive rate significantly.

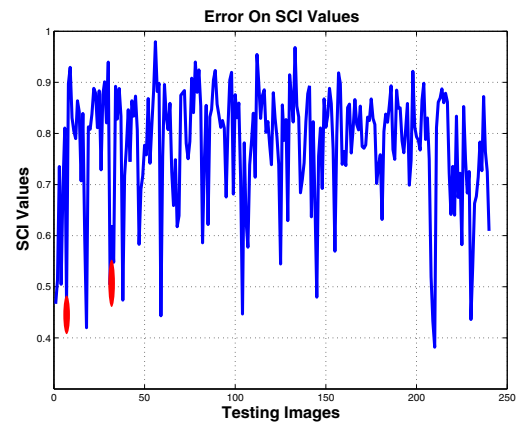

Fig. 8. Plot of the SCI Values of test images of a random trial of the ND dataset. Red dots indicate the wrongly classified images. Observe that the wrongly classified images have very low SCI values and hence the corresponding vectors are not sparse.

\section{CONCLUSiOn AND Future Work}

In this paper, we have developed a unified approach for iris image selection and recognition. Results of simulation and real data indicate that our method can handle a wide variety of commonly possible distortions in iris image acquisition including blur, occlusions and segmentation errors, and give high recognition results. Currently we are working on extending our algorithm for automatic recognition of iris videos in unconstrained conditions without full cooperation of the subjects. This is a major challenge in our grand goal of developing iris recognition systems in surveillance applications with limited cooperation from the subjects. We are also exploring ways of developing a minimal set of training images from the incoming video, which can span the testing image space and possibility of adapting other algorithms such as elastic net [18]. A method that can handle poor quality iris images using compressed sensing techniques [19], [20] is also being investigated.

\section{REFERENCES}

[1] K. W. Bowyer and P. J. Flynn,’The ND-IRIS-0405 Iris Image Dataset“, Notre Dame CVRL Technical Report.

[2] J. Wright, A. Y. Yang, A. Ganesh, S. S. Sastry, and Y. Ma, "Robust Face Recognition via Sparse Representation," IEEE Trans. Pattern Anal. Mach. Intell., accepted for future publication

[3] J. Daugman, "How iris recognition works," IEEE Trans. Circ. Syst.Video Technol., vol.14, no.1, pp.21-30, 2004.

[4] R. P. Wildes, "Iris recognition: An emerging biometric technology," Proc. IEEE vol. 85, no. 9, pp. 1348-1363, 1997.

[5] K. W. Bowyer, K. Hollingsworth, and P. J. Flynn, "Image understanding for iris biometrics: A survey", Computer Vision and Image Understanding, vol. 110, no. 2, pp.281-307, 2008.

[6] M. Gamassi, M. Lazzaroni, M. Misino, V. Piuri, D. Sana, and F. Scotti, "Quality assessment of biometric systems: a comprehensive perspective based on accuracy and performance measurement," Instrumentation and Measurement, IEEE Transactions on, vol. 54, pp. 1489-1496, 2005.

[7] H. Proenca, L. A. Alexandre, "A method for the identification of noisy regions in normalized iris images," in: International Conference on Pattern Recognition, pp. 405-408, August 2006.

[8] X.-D. Zhu, Y.-N. Liu, X. Ming, and Q.-1. Cui, "Quality Evaluation Method of Iris Images Sequence Based on Wavelet Coefficients in Region of Interest", presented at The Fourth International Conference on Computer and Information Technology (CIT), 2004.

[9] L. Ma, T. Tan,Y. Wang,D. Zhang, "Personal Identification Based on Iris Texture Analysis",IEEE Transactions on Pattern Analysis and Machine Intelligence, vol. 25 , no. 12, pp. 1519-1533, 2003

[10] S. Rakshit, D. Monro, "Iris image selection and localization based on analysis of specular reflection", IEEE Workshop on Signal Processing Applications for Public Security and Forensics, SAFE, pp 1-4, April 2007.

[11] E. Krischen, S. Garcia-Salicetti, B. Dorizzi, "A new probabilistic Iris Quality Measure for comprehensive noise detection", IEEE First International Conference on Biometrics:Theory, Applications and Systems (BTAS 2007), Washington, USA, September 2007.

[12] Y. Chen, S. Dass, A. Jain, "A Localized Iris Image Quality Measure Using 2D Wavelets",Proc. Intl. Conference on Biometrics, Hongkong, China, Jan 6-8, 2006.

[13] J. R. Matey, O. Naroditsky, K. Hanna, R. Kolczynski, D. LoIacono, S. Mangru, M. Tinker, T. Zappia, W. Y. Zhao, "Iris on the Move ${ }^{T M}$ : acquisition of images for iris recognition in less constrained environments", Proc. IEEE, vol. 94, no. 11, pp. 1936-1946, 2006.

[14] F. W. Wheeler, A. G. Perera, G. Abramovich, B. Yu, P. H. Tu, "Standoff Iris Recognition System", IEEE Second International Conference on Biometrics: Theory, Applications and Systems (BATS), 2008.

[15] S. Chen, D. Donoho, and M. Saunders, " Atomic Decomposition by Basis Pursuit," SIAM J. Sci. Comp., vol. 20, no. 1, pp. 33-61, 1998.

[16] L. Masek, P. Kovesi, "MATLAB Source Code for a Biometric Identification System Based on Iris Patterns", The School of Computer Science and Software Engineering, The University of Western Australia. 2003.

[17] E. van den Berg and M. P. Friedlander, "Probing the Pareto frontier for basis pursuit solutions", SIAM J. Sci. Comp, vol. 31, no. 2, pp. 890-912, 2008

[18] H. Zou and T. Hastie, "Regularization and Variable Selection via the Elastic Net," Journal of the Royal Statistical Society, vol. 67, no. 2, pp. 301-320, 2005.

[19] D. Donoho, "Compressed sensing," IEEE Trans. Info. Theory, vol. 52, no. 4, pp. 1289-1306, April 2006.

[20] E. Candes, J. Romberg and T. Tao, "Robust Uncertainty Principles: Exact Signal Reconstruction from Highly incomplete Frequency Information," IEEE Trans. Info. Theory, vol. 52, no. 2, pp. 489-509, Feb. 2006. 